\title{
MICRO-BUNCHING OF THE AGS SLOW EXTRACTED BEAM FOR A RARE KAON DECAY SEARCH
}

\author{
J. Glenn, M. Sivertz, I. Chiang, D. Lazarus, Brookhaven National Laboratory*, Upton N.Y., U.S.A. \\ S. Koscielniak, TRIUMF ${ }^{\dagger}$, Vancouver, B.C., Canada
}

\section{Abstract}

The AGS Slow Extracted Beam (SEB) must be chopped with 250 ps bursts every $40 \mathrm{~ns}$ to permit time-of-flight (ToF) measurement of the secondary $K^{0}$ beam. Standard techniques to produce this level of bunching would require excessive rf voltage, thus we have developed a "MicroBunching" technique of extracting the beam as it is forced between empty rf buckets. A specification of the required rf system will be given. Four-dimensional model simulations of particle dynamics for the planned rf and extrac-tion systems will be shown. Simulations of previous tests along with the test measurements are also presented. Measurement of tight bunching requires dedicated instrumentation. The design of a detector system to measure bunch widths and the extinction factor between bunches will be given; considerations include the various particles produced and transported, timing precision and background.

\section{INTRODUCTION}

In the BNL AGS-2000 era, it is proposed[1] to search for the CP violating decay $K_{L} \rightarrow \pi^{0} \nu \bar{\nu}$ competing with the background $K_{L} \rightarrow \pi^{0} \pi^{0}$. To better determine the branching ratio, the single $\pi^{0}$ will be reconstructed from photon decays and the $K_{L}$ momentum will be determined event-byevent. The ToF technique is only recently feasible at the AGS[2]. In micro-bunching[3], a quasi-coasting (i.e. debunched) beam is forced (by ramping down the main magnet field) between empty rf buckets that are centred near the extraction radius. The betatron tune depends on the longitudinal momentum; and chromatic extraction is effected by a $\frac{1}{3}$-integer transverse resonance driven by sextapoles. The farther off-momentum particles, which cluster about the astable fixed point, are preferentially extracted. The method has been tested at the AGS with a $20 \mathrm{kV}, 93 \mathrm{MHz}$ cavity yielding proton bunches of $0.6 \mathrm{~ns}$ length and $11 \mathrm{~ns}$ spacing. However, the $40 \mathrm{~ns}$ bunch spacing for ToF implies the use of a $25 \mathrm{MHz}$ rf system, and the $0.25 \mathrm{~ns}$ r.m.s. deviation at this lower frequency is more challenging.

When large circulating beam current is involved (a spill of $\approx 10^{14}$ protons each $3 \mathrm{~s}$ ) the micro-bunching scheme has the advantage that the charge density and Fourier components of a coasting beam are an order of magnitude smaller than for bunched beam extraction. Consequently, transverse tune shift and spread, cavity beam-loading and other longitudinal wake-fields are all reduced.

\footnotetext{
* Work performed under the auspices of the US Dept. of Energy.

${ }^{\dagger}$ Also supported via a contribution through the NRC of Canada.
}

\section{SIMULATIONS}

Previous simulations[2, 4] treated the longitudinal and transverse phase spaces separately; and because the momentum width of the resonance was assumed infinitesimal, these simulations underestimated the extracted bunch length. A second generation simulation, based on a marriage of the SLEX[5] and LONG1D[6] programs, models both phase planes and the coupling between them. Let $\Delta E, \Delta p$ be energy and momentum deviation, and $\phi \mathrm{rf}$ phase. Subscript $s$ denotes synchronous orbit. The longitudinal motion is modelled by

$$
\begin{gathered}
d(\Delta E) / d t=\left(\beta c e / 2 \pi R_{s}\right)\left[V(\phi)-2 \pi \rho_{0} R_{s} \dot{B}\right] \\
\frac{d}{d t} \theta=\frac{\beta_{s} c}{R_{s}} \eta \frac{\Delta p}{p_{s}} \text { and } \frac{d \phi}{d t}=h \frac{d \theta}{d t}+\Delta \omega(t) .
\end{gathered}
$$

Let $\alpha$ and $\beta$ be Twiss values. The transverse (i.e. horizontal) motion is derived from the hamiltonian[7]

$$
H(Q, P)=\frac{1}{2} \delta \nu_{i}(t, \Delta p)\left[Q^{2}+P^{2}\right]+A(t)\left[3 P Q^{2}-P^{3}\right]
$$

The coordinates $Q, P$ are taken in a frame rotating with tune $\Delta \nu=\nu-m / 3$. In this frame, the particles are brought approximately to rest; there is a small residual rotation $\delta \nu_{i}$, and some amplitude growth due to the non-linear drive terms. $A$ is the Fourier component of the sextapole strength around the ring. $Q, P$ are related to the position and divergence $x, x^{\prime}$ via first the transformation (4) and then one or more rotations so as to give a separatrix which is an upright triangle.

$$
\left[\begin{array}{l}
Q \\
P
\end{array}\right]=\frac{1}{\sqrt{\beta}}\left[\begin{array}{ll}
1 & 0 \\
\alpha & \beta
\end{array}\right]\left[\begin{array}{c}
x \\
x^{\prime}
\end{array}\right]
$$

Resonance width The triangular separatrix with area equal to the transverse emittance $\varepsilon_{H}$ has vertices $\left[ \pm Q_{0},-P_{0}\right]$ and $\left[0,+2 P_{0}\right]$ where $Q_{0}= \pm\left(\pi \varepsilon_{H} / \sqrt{3}\right)^{1 / 2}$ and $P_{0}=Q_{0} / \sqrt{3}$. Here we take $Q_{0}$ positive (negative) if the nominal tune is above (below) the integral resonance. Particles with zero betatron amplitude come into resonance when $\delta \nu_{i}=0$. Particles with betatron amplitudes touching the separatrix come into resonance when their $\left|\delta \nu_{i}\right| \leq 6 P_{0} A \equiv \delta \nu_{w}$.

Because of the longitudinal motion the betatron tune varies, carrying the particles toward resonance. Usually in SEB schemes the condition $\epsilon_{H} \gg|\xi \Delta \dot{p}| /\left(p_{s} A^{2} \pi 6 \sqrt{3}\right)$ is satisfied and there is sufficient time for the amplitude to grow large enough for extraction at the septum, and in this case the momentum width is $\Delta p_{w} / p_{s}=\delta \nu_{w} / \xi$. However, 
the gap between neighbour rf buckets acts as a venturi; and so the narrower the constriction in rf phase, the faster is the motion in $\Delta p$. When it is fast enough, particles pass through the betatron resonance and reverse the sign of $\delta \nu_{i}$. Hence the width of the resonance may rise to $2 \Delta p_{w}$.

Central momentum of bucket The betatron resonance is located at $\Delta p_{\text {res }} / p_{s}=\Delta \nu / \xi$. Where should one place the central momentum of the rf bucket? If the venturi is wide and $d \Delta p / d t$ is slow, then the bucket may be centred at $\Delta p_{\text {res }}$ and the momentum width will be $\Delta p_{w}$ and the rf phase-extent of extracted bunches will be the minimum possible. If the venturi is narrow and $d \Delta p / d t$ is fast, and the bucket is centred at $\Delta p_{\text {res }}$, the momentum width will be $2 \Delta p_{w}$ and some particles may evade extraction. In this case, the centre should be moved slightly above the resonance so that extraction can benefit from the slower movement in $\Delta p$; this guarantees that particles are extracted, but increases (typically doubles) the rf phase-extent.

Effect of chromaticity The larger the modulus of chromaticity, the smaller is the momentum width of the betatron resonance; which is immediately beneficial to bunch length. The smaller momentum range also implies that the magnet field ramp required to sweep the entire beam across the resonance is smaller. The lowering of $|d B / d t|$ reduces the synchronous phase and narrows the gaps between $\mathrm{rf}$ buckets, which is also beneficial.

Higher harmonics A cavity with an even harmonic antiphased to the fundamental may be used to distort and stretch the rf buckets so as to further shorten the bunches.

\subsection{Results \& rf requirements}

\begin{tabular}{|c|c|c|c|c|c|c|c|}
\hline $\begin{array}{c}-\dot{B} \\
(\mathrm{~T} / m \mathrm{~s})\end{array}$ & $\begin{array}{c}V_{1} \\
(\mathrm{kV})\end{array}$ & $\begin{array}{c}V_{h} \\
(\mathrm{kV})\end{array}$ & $\begin{array}{c}\Delta p / p \\
\%\end{array}$ & $\begin{array}{c}66 \% \\
(\mathrm{~ns})\end{array}$ & $\begin{array}{c}90 \% \\
(\mathrm{~ns})\end{array}$ & $\begin{array}{c}99 \% \\
(\mathrm{~ns})\end{array}$ & $\begin{array}{c}\text { cross } \\
\%\end{array}$ \\
\hline .773 & 100. & 0.0 & 0.46 & 4.26 & 10.7 & 17. & 1.01 \\
.773 & 100. & 0.0 & 0.53 & 1.35 & 2.09 & 3.50 & 1.70 \\
.773 & 100. & 0.0 & 0.60 & 2.51 & 4.66 & 8.41 & .187 \\
\hline .257 & 100. & 100. & 0.53 & 0.67 & 1.20 & 2.12 & 2.97 \\
.257 & 150. & 150. & 0.53 & 0.67 & 1.19 & 2.12 & 3.64 \\
.257 & 150. & 150. & 0.46 & 3.16 & 5.21 & 7.7 & 0.70 \\
.257 & 150. & 150. & 0.50 & 0.92 & 2.47 & 4.63 & 0.90 \\
.257 & 150. & 150. & 0.60 & 1.18 & 2.60 & 4.63 & .219 \\
.257 & 150. & 0.0 & 0.60 & 1.82 & 3.25 & 6.09 & .050 \\
.257 & 300. & 0.0 & 0.60 & 1.81 & 3.22 & 5.95 & .224 \\
\hline .129 & 150. & 150. & 0.23 & 1.35 & 2.80 & 4.14 & 5.2 \\
.129 & 150. & 150. & 0.30 & .855 & 1.59 & 2.59 & 1.78 \\
.129 & 150. & 150. & .264 & .355 & .647 & 1.16 & 7.0 \\
\hline .129 & 150. & 300. & .264 & .322 & .580 & 1.04 & 13.6 \\
.129 & 150. & 300. & 0.30 & .644 & 1.29 & 2.18 & 4.24 \\
\hline
\end{tabular}

A variety of schemes has been simulated with various single and dual harmonic $(h=2,4,6)$ voltages, and various $\dot{B}$ and chromaticity. A summary of results is tabulated above. "Cross" indicates the fraction which pass through the resonance and $\Delta p$ denotes the bucket central momentum. The presently preferred scheme uses fundamental and $4^{\text {th }}$ harmonic with $150 \mathrm{kV}$ on each cavity. The figures 1 3 below demonstrate how the resonance crossing (and $\Delta p$ width) is reduced and the bunch length increased by moving the bucket centre above the resonance centre (blue line). Resonance width is shown by brown/coral lines. Left,right sub-figures are the cases $\Delta p / p=0.53,0.6$ respectively.
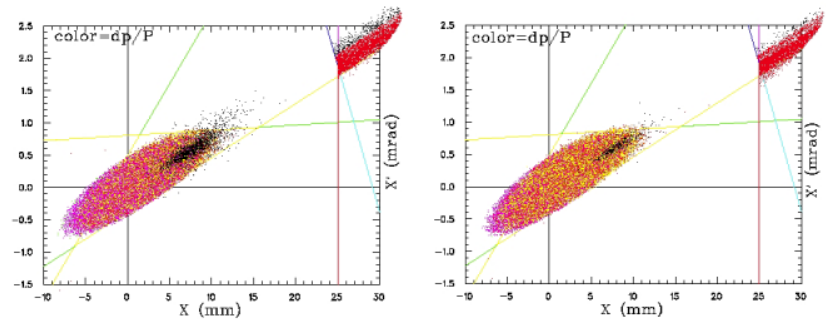

Figure 1: Transverse phase spaces.
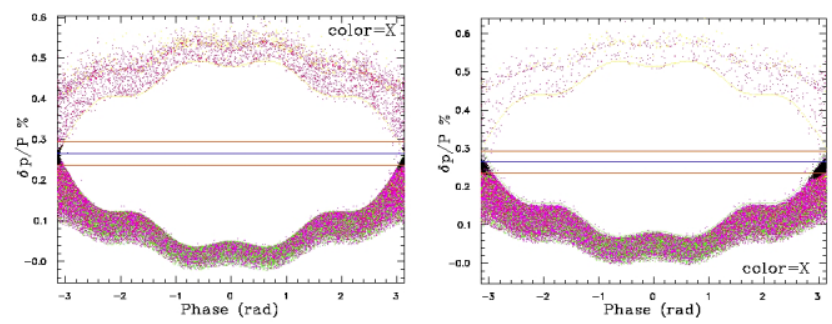

Figure 2: Longitudinal phase spaces.
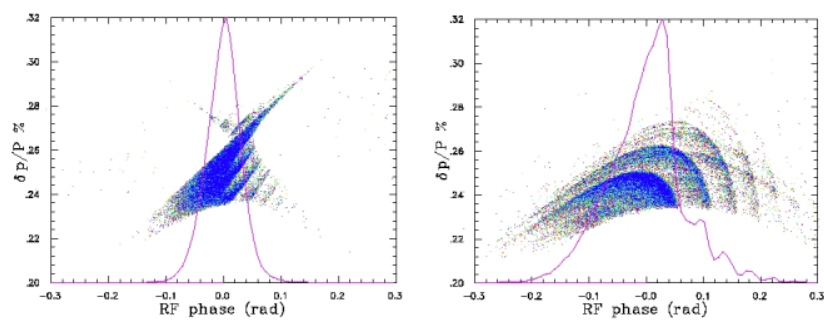

Figure 3: Microbunch shapes.

Due to the long time span, c.p.u. requirement was reduced by tracking only the upper $\frac{1}{3}$ of the beam in $\Delta p / p$ $\left(5 \times 10^{4}\right.$ macro-particles) for 1 second $\left(\approx 3.3 \times 10^{5}\right.$ turns $)$.

\section{PREVIOUS TESTS AND DATA}

Several exploratory measurements were made in 1997 with protons and gold ions using a $93 \mathrm{MHz}$ cavity at $20 \mathrm{kV}$. The proton beam results were reported in Ref.[2]. Results for the much lower intensity Au beam are reported here. The extracted gold ions were run into a 5 micron thick quartz radiator. Two phototubes monitored čerenkov light from the quartz and started a $40 \mathrm{GHz}$ TDC. The stop pulse came from a PLL on the rf voltage. The fast electronics were in a Camac crate controlled by a PC which made the data available for off-line evaluation.

Figures 4,5 show example spills lasting $1.5 \mathrm{sec}$ and with $\dot{B} / B=0.33 \% / \mathrm{sec}$. The time delay and width of the extracted bunch vary with time in response to several factors: e.g. the frequency and slew rate of the rf which alter the position and shape of the bucket with respect to the resonance; also the extraction servo-loop, which varies $\dot{B}$ so as to make the spill more uniform, is particularly active toward the end.

The small Au transverse emittance $\left(\epsilon_{H}^{*} \approx 15 \pi \mathrm{mm} . \mathrm{mr}\right)$ leads to a narrow resonance width $(\Delta p / p \approx \pm 0.025 \%)$ and 
combined with the small height of the buckets $(\Delta p / p \approx$ $\pm 0.035 \%$ ) scanning of the rf to explore optimum conditions is a delicate procedure requiring small increments. The average time delay (or phase) and the delay width are plotted versus frequency offset; the zero was chosen to match minima in width. Figure 6 summarizes the experimental results. Values for the entire spill and for a "cleaner" subset $(\approx 300 \mathrm{~ms})$ are given. Simulation results for a much broader frequency scan are given in figure 7 . Over the range for which comparison can be made, there is agreement on the broad trends but not on the details.

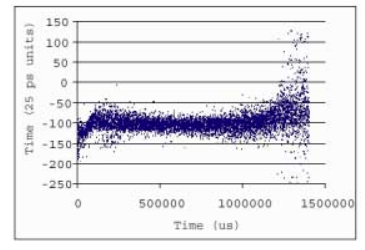

Figure 4: SEB Spill, $\Delta f=-196 \mathrm{~Hz}$

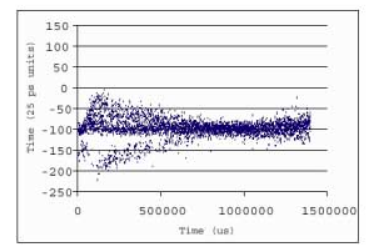

Figure 5: SEB Spill, $\Delta f=-11 \mathrm{~Hz}$

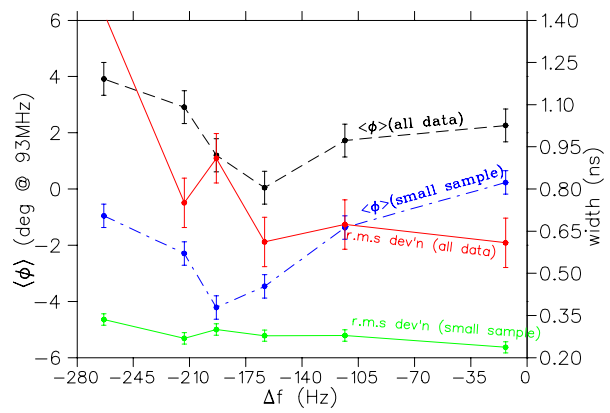

Figure 6: Gold ion experiments summary

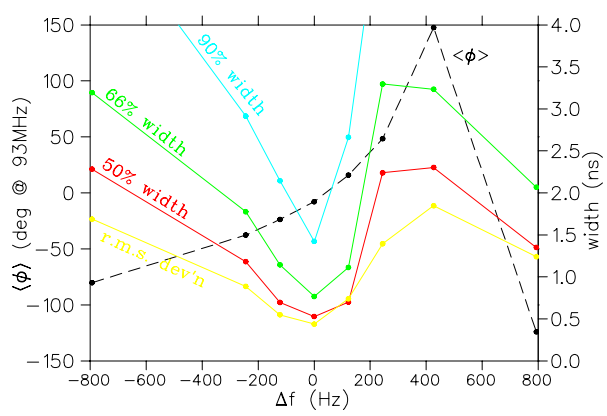

Figure 7: Gold ion simulations summary

\section{DETECTOR SYSTEM DESIGN}

To monitor the micro-bunch time structure, we plan to build a gamma-ray telescope which will observe the photons produced, primarily by $\pi^{0}$ decay, in the target. The photons are prompt, thus duplicating the time structure of the proton beam. Slower charged particles with momenta less than $2 \mathrm{GeV} / \mathrm{c}$ will be removed with a sweeper magnet.

The detector consists of lead converter, to initiate the photon shower, followed by a pair of small beam-defining scintillators. Immediately after them, a lead tungstate calorimeter will measure the photon energy. The telescope is aligned in the horizontal plane of the beamline, at $135^{\circ}$ from the incident proton direction. The trigger from the detector will be the coincidence of the scintillators and the calorimeter above threshold. To veto any high-momentum charged particles that pass through the sweeper magnet, an additional scintillator upstream of the lead converter will be used in anticoincidence with the trigger.

The trigger rate will be tuned by adjusting the size and the overlap of the two small scintillators. We would like to have a counting rate high enough to give nearly instant feedback to the rf systems controlling the bunch structure. We anticipate photon counting rates in the range of 0.1 to 1.0 MHz. We will also use the detector to measure the interbunch extinction which is required to be $\leq 10^{-3}$ of the microbunch intensity.

The number of neutrons accompanying the photons is expected to be large; most will pass through the lead converter and the two small scintillators without depositing significant energy. Those that do make a track will fail to give a signal above the energy threshold in the calorimeter.

The phototube signals will be discriminated, and the output pulses will be used to form a coincidence for the trigger, and for a TDC-Stop pulse. The TDC-Start will be produced by a signal from the rf timing system. The distribution of timing pulses will give a time profile of the proton beam. The number of photons counted will give a measure of the beam intensity.

\section{CONCLUSION}

We have reported slow extraction simulations with a new 4D computer program which suggest the novel occurrence of betatron resonance crossing when $d(\Delta p) / d t$ is large enough. The effect can be regulated by adjusting the relative $\Delta p$ offset of the bucket and resonance. We have reported some previous measurements with gold ions; unfortunately the program is not entirely validated by these results. Finally we have proposed a micro-bunch monitor for real-time control of the beam structure. We acknowledge the initial assistance of U. Wienands with SLEX.

\section{REFERENCES}

[1] L. Littenberg \& J. Sandweiss (editors): AGS-2000, Experiments for the 21st Century, Proc. of the workshop held at B.N.L. May 1996. BNL-52512.

[2] J.W. Glenn et al.: Micro-bunching the AGS slow external beam, Proc. of 1997 Particle Accelerator Conf. Vancouver, B.C. Canada, May 1997, IEEE Press, pg. 967.

[3] R. Cappi \& Ch. Steinbach: Low frequency duty factor improvement for the CERN PS slow extraction..., IEEE Trans. Nucl. Sci., Vol. NS-28, No.3, 1981, pg. 2806.

[4] S. Koscielniak: Longitudinal Simulations of $25 \mathrm{MHz}$ MicroBunching in the BNL AGS, TRI-DN-01-01, January 2001.

[5] U. Wienands \& R. Servranckx: Towards a slow extraction system.., Proc. $1^{\text {st }}$ EPAC, Rome, June 1988, pg. 269.

[6] S. Koscielniak: Longld user's guide, TRI-DN-97-12

[7] K.R. Symon: Extraction at third-integral resonance, parts I,II,II; Fermilab Notes FN-130, FN-134, FN-140, April 1968. 